\title{
Ações do programa de puerpério na atenção primária: uma revisão integrativa
}

\author{
Postpartum program actions in primary health care: \\ an integrative review
}

Tatiane Baratieri (https://orcid.org/0000-0002-0270-6395) ${ }^{1}$

Sonia Natal (https://orcid.org/0000-0001-6155-4785) ${ }^{1}$

${ }^{1}$ Universidade Federal de Santa Catarina. R. Eng. Agronômico Andrei Cristian Ferreira s/n, Trindade. 88040-900 Florianópolis SC Brasil.

baratieri.tatiane@gmail.com

\begin{abstract}
Puerperium is a period of significant morbimortality for women, and Primary Health Care (PHC) is important in developing actions to meet women's health needs. This study aimed to systematize the knowledge produced on postpartum care programs actions within PHC at both national and international levels. This is an integrative review of the literature in databases LILACS (Latin American and Caribbean Health Sciences Literature), BDENF (Nursing Database), SciELO (Scientific Electronic Library Online) and PubMed (US National Library of Medicine). Search was performed in the period April-May 2017. Forty-three papers met the selection criteria. Results indicate that PHC has the physical structure to provide puerperae with care, but has a shortage of human and material resources; there is low postpartum consultation coverage and home visits; there is a good evaluation of the incentive for breastfeeding, but focused on the child; international screening of Postpartum Depression through the Edinburgh Postnatal Depression Scale and care shortage for this condition in Brazil. Postpartum care still focuses on care for the newborn and is mostly restricted to the immediate and late puerperium.
\end{abstract}

Key words Primary health care, Postpartum, Women's health
Resumo O puerpério trata-se de um período de significativa morbimortalidade para as mulheres, e a Atenção Primária à saúde (APS) é importante no desenvolvimento de ações para atender as necessidades de saúde das mulheres. Objetivouse sistematizar o conhecimento produzido sobre as ações de programas de atenção pós-parto no âmbito da APS, tanto em nível nacional, como internacional. Utilizou-se revisão integrativa de literatura de artigos junto às bases Lilacs (Literatura Latino-Americana e do Caribe em Ciências da Saúde), BDENF (Base de dados em Enfermagem), SciELO (Scientific Electronic Library Online) e PubMed (Biblioteca Nacional de Medicina dos Estados Unidos). A busca ocorreu de abril a maio de 2017. Atenderam aos critérios de seleção 43 artigos. Os resultados apontam que: a APS possui estrutura física para atenção à puérpera, porém com déficit em recursos humanos e materiais; há baixa cobertura de consulta pós-parto e visita domiciliar; boa avaliação do incentivo ao aleitamento materno, porém com foco na criança; rastreamento da Depressão Pós-Parto internacionalmente por meio da "Edimburgh Post-Natal Depression Scale", e déficit na atenção a esse agravo no Brasil. A atenção pós-parto ainda tem como foco o cuidado ao recém-nascido e são restritos, em sua maioria, ao puerpério imediato e tardio.

Palavras- chave Atenção primária à saúde, Período pós-parto, Saúde da mulher 


\section{Introdução}

Embora a expectativa de vida seja maior para as mulheres do que para os homens na maioria dos países, uma série de fatores sociais e de saúde se combinam acarretando menor qualidade de vida para as mulheres ${ }^{1}$. A desigualdade de gênero conduz a muitos riscos para a saúde das mulheres, incluindo violência física e sexual, infecções sexualmente transmissíveis, malária, doença pulmonar obstrutiva crônica e morbimortalidade materna ${ }^{1}$.

As taxas de mortalidade durante a gravidez e o parto permanecem elevadas nos países em desenvolvimento, apesar do seu decréscimo mundial nas últimas décadas, de modo que $99 \%$ dos óbitos maternos ocorrem nesses países ${ }^{1}$. Óbitos evitáveis continuam ocorrendo em proporções alarmantes, sobretudo decorrente de doenças hipertensivas, hemorragias, sepse/infecções e complicações relacionadas ao aborto ${ }^{1,2}$.

A saúde materna é considerada sensível à Atenção Primária à Saúde (APS) ${ }^{2,3}$, portanto, aumentar a qualidade desse ponto de atenção é fundamental para reduzir as taxas de mortalidade dessa população. Óbitos e morbidades que ocorrem durante a gravidez, parto e puerpério são passíveis de serem evitadas com a implementação de ações integradas e de acesso universal, por meio de tecnologias leves e cuidados primários ${ }^{2,3}$.

Ressalta-se que a maioria dos óbitos maternos se concentra no puerpério imediato $\left(1^{\circ}\right.$ ao $10^{\circ}$ dia pós-parto), além de ser considerado uma fase de morbidade significativa ${ }^{4,5}$ que se estende para o puerpério tardio (do $11^{\circ}$ ao $45^{\circ}$ dia) e remoto (após $\left.45^{\circ} \mathrm{dia}\right)^{6}$, com estudos que apontam para agravos persistindo por mais de 4 anos pós-parto ${ }^{7,8}$. A APS é a principal responsável pela atenção à mulher no pós-parto, por meio da integração do conhecimento técnico e a capacidade de acolher, apoiar e detectar mudanças físicas e emocionais precocemente, realizar prevenção, tratamento e acompanhamento da mulher, com encaminhamento para outros serviços quando necessário ${ }^{9,10}$.

Considerando a significativa morbimortalidade das mulheres no período puerperal, e o fato da APS ser a ordenadora do cuidado e responsável pela resolução da maioria dos problemas de saúde, destaca-se a importância do desenvolvimento de ações efetivas e eficazes a fim de atender as necessidades de saúde das mulheres no puerpério.

Para tanto, as políticas de saúde em âmbito nacional e internacional envidam esforços e estabelecem diretrizes, ações e estratégias para atenção puerperal, em especial em cuidados primários, na compreensão de que recursos adequados são requisitos fundamentais para atenção integral ${ }^{1,6}$. Apesar dessas iniciativas, há evidências de que o cuidado pós-parto na APS necessita adequações, com melhora da estrutura física e material, gestão e assistência nos serviços de saúde, qualificação profissional, cuidado centrado na mulher, superação da atenção tecnicista, contribuindo assim para a melhoria da saúde da mulher ${ }^{7,10-12}$.

Conhecer as atividades desenvolvidas e resultados alcançados pelos programas de atenção puerperal é importante para o planejamento e melhoria do cuidado na APS voltado para a saúde da mulher no pós-parto. Com base no exposto, questiona-se: Quais são as características e resultados dos estudos que tratam da atenção pós-parto no âmbito da APS? Assim, o estudo objetivou sistematizar o conhecimento produzido sobre a atenção pós-parto no âmbito da APS, tanto em nível nacional, como internacional.

\section{Metodologia}

O presente estudo é uma revisão integrativa da literatura, que se caracteriza por um método que permite a busca, avaliação crítica e síntese de evidências disponíveis acerca do tema investigado, em seu produto final se constitui do estado atual do conhecimento, implementação de intervenções e identificação de lacunas que norteiam o desenvolvimento de outros estudos ${ }^{13}$.

As etapas operacionais da revisão integrativa são: identificação do tema e seleção da hipótese ou questão de pesquisa; estabelecimento de critérios de inclusão e exclusão de estudos/ amostragem ou busca na literatura; definição das informações a serem extraídas dos estudos selecionados/categorização dos estudos; avaliação dos estudos incluídos na revisão integrativa; interpretação dos resultados; e apresentação da revisão/síntese do conhecimento ${ }^{13}$.

Na primeira etapa foi formulada a seguinte questão norteadora: Quais são as características tipo de estudo, critérios, parâmetros, indicadores - e resultados dos estudos que tratam da atenção pós-parto no âmbito da Atenção Primária à Saúde?

$\mathrm{Na}$ segunda etapa foi realizada a busca das referências por meio da Biblioteca Virtual em Saúde (BVS), que faz busca simultânea nas principais bases de dados de amplitude nacional e 
internacional, sendo selecionados pelo filtro as bases Lilacs (Literatura Latino-Americana e do Caribe em Ciências da Saúde e BDENF (Base de dados em Enfermagem). Também foi realizada busca avançada na SciELO (Scientific Electronic Library Online) e PubMed (Biblioteca Nacional de Medicina dos Estados Unidos).

Quanto aos critérios de inclusão para seleção dos artigos foram estabelecidos: ser artigo original, responder à questão norteadora; ser publicado nos idiomas português, inglês ou espanhol; estar publicado em revista científica com Qualis B2 no mínimo para saúde coletiva; e quando não disponível a avaliação pelo Qualis, foi verificado o JCR (Journals Impact Factors), incluindo artigos com no mínimo 1,0. Foram excluídos estudos repetidos em uma ou mais base de dados.

A busca das referências ocorreu entre os meses de abril e maio de 2017 utilizando-se os seguintes descritores indexados nos Descritores em Ciências da Saúde (DeCS), os quais foram todos agrupados utilizando-se as expressões boleanas AND e OR: período pós-parto OR postpartum period OR período pós-parto OR saúde materna OR maternal health OR salud materna OR saúde da mulher OR women's health OR salud de la mujer OR saúde materno-infantil OR maternal and child health OR salud materno infantil AND Atenção Primária à Saúde OR Primary Health Care OR Atención Primaria de Salud OR Saúde da Família OR Family Health OR Salud de la família. Na base de dados PubMed foram utilizados os Mesh Terms: postpartum period OR maternal health OR women's health AND primary health care OR family health.

Justifica-se a ampla gama de descritores utilizados já que o assunto pós-parto é tratado em conjunto com outros relacionados à saúde da mulher, e a restrição ao descritor "postpartum period" limitaria a possibilidade de encontrar estudos que abordam o tema. Dada a relevância de alguns estudos, selecionou-se referências que contemplavam os critérios de inclusão do estudo citadas pelos artigos selecionados.

A fim de avaliar a qualidade metodológica dos estudos encontrados, foram utilizados instrumentos específicos para cada tipo de estudo. Os estudos avaliativos foram analisados por meio da adaptação dos critérios da metaavaliação estabelecidos por Stufflebeam ${ }^{14}$, sendo excluídos estudos com classificação "fraco" e "razoável”, ou seja, com mais de $50 \%$ das questões de avaliação respondidas negativamente. Os demais estudos foram avaliados utilizando-se o Critical Appraisal Skills Programme (CASP) ${ }^{15}$, que se constitui em check lists específicos para cada tipo de estudo, e não sugere um sistema de pontuação, sendo assim os autores optaram por utilizar a mesma classificação de Stufflebeam ${ }^{14}$, sendo excluídos estudos razoáveis e ruins. Esses estudos, exceto os avaliativos, ainda foram classificados quanto ao seu nível de evidência, de acordo com os critérios de Stetler et al. ${ }^{16}$.

Para a realização da terceira etapa (definição das informações a serem extraídas dos estudos selecionados) foi utilizado o instrumento elaborado por $\mathrm{Ursi}^{17}$ que aborda, dentre outras informações, a identificação do estudo, características metodológicas, avaliação do rigor metodológico, intervenções e resultados encontrados.

Os resultados dos estudos selecionados foram sistematizados segundo semelhanças dos dados analisados em cada pesquisa. A síntese e interpretação dos dados foram fundamentadas nos resultados da avaliação crítica dos estudos selecionados. Foi realizada a comparação com o conhecimento teórico, identificação de conclusões e implicações resultantes da revisão integrativa.

A fim de minimizar viéses, a busca, avaliação e seleção dos estudos se deu por dois revisores, e ao final realizada discussão de consenso sobre os artigos a serem incluídos na revisão.

\section{Resultados}

Após realizar os cruzamentos entre os DeCS/ Mesh Terms foram encontrados 1974 referências e, ao término das estratégias de seleção do material, selecionou-se 43 para análise e discussão, conforme apresentado na Figura 1.

Quanto ao ano de publicação dos artigos variou de 1995 a 2016, com a maioria, 69,8\% (30), dos últimos cinco anos, sendo 20,9\% (9) publicados em 2013. Sobre o local do estudo, 60,5\% (26) foram realizados no continente Americano, a maioria 51,2\% (22) no Brasil. Outros estudos se deram nos continentes europeu, 6 (14\%), Oceania, 6 (13,9\%), Ásia, 2 (4,7\%), África 2 (4,7\%), e um em três países, Bangladesch (Ásia), Nepal (Ásia) e Malawi (África). O idioma predominante foi o inglês, $51,2 \%$ (22), seguido do português, 44,2\% (19), e 4,7\% (2) espanhol.

A maioria dos estudos, 76,7\%(33) seguiu a abordagem quantitativa, e quanto ao tipo de estudo 37,2\% (16) são avaliativos, 20,9\% (9) qualitativos, $13,9 \%$ (6) transversais, $11,6 \%$ (5) ensaio clínico, 7\% (3) coorte e o mesmo número quase experimental, e um descritivo. Sobre a avaliação da qualidade metodológica: dos 16 artigos ava- 


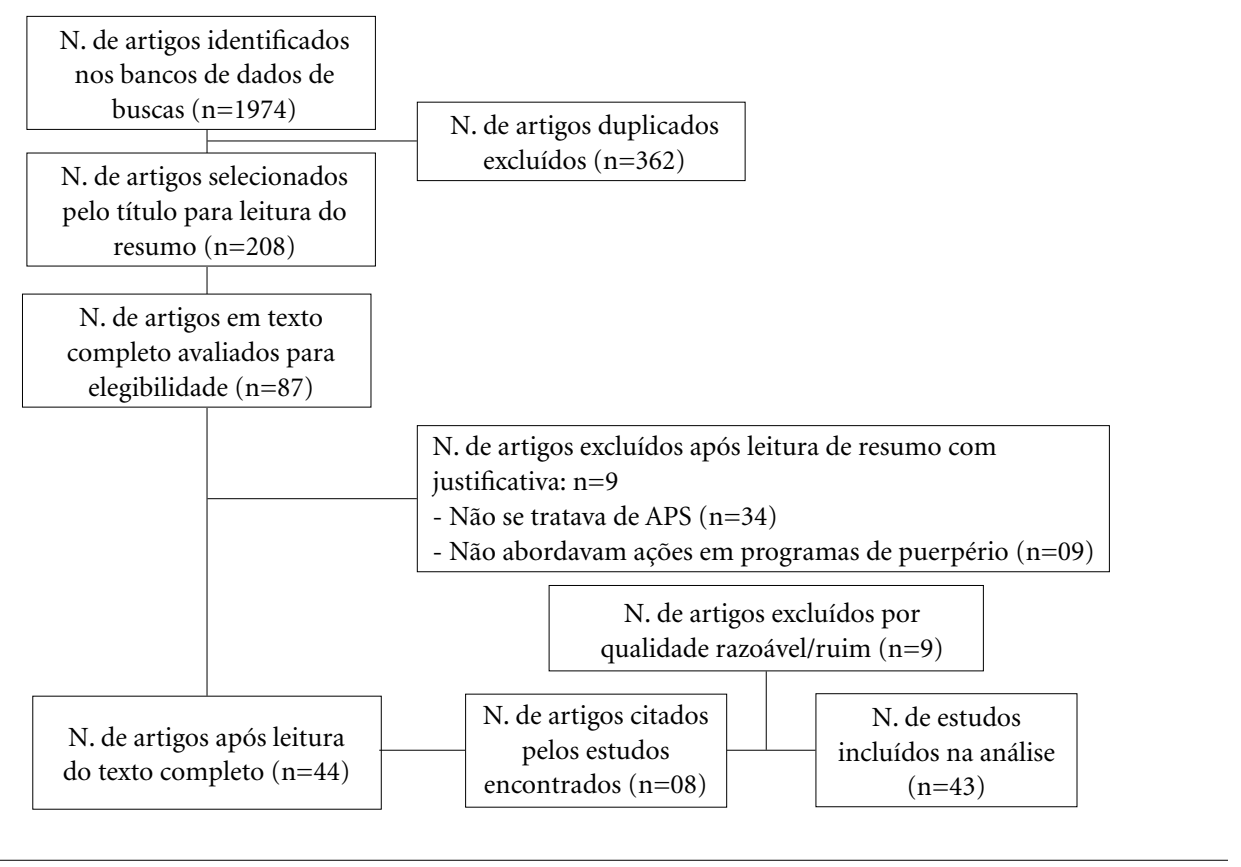

Figura 1. Fluxograma relacionado ao processo de seleção dos artigos conforme PRISMA/2015.

liativos, $12,5 \%$ (2) foram avaliados como excelente, $56,25 \%$ (9) muito bom e $31,25 \%$ (5) bom, com emissão julgamento de valor em dois ${ }^{18,19}$. Os demais foram classificados quanto ao nível de evidência, predominando o nível IV, 59,3\% (16), seguido do nível III, 22,2\% (6) e nível II, 18,5\% (5); e quanto ao CASP, a maioria foi classificado como muito bom, $51,8 \%$ (14), $25,9 \%$ (7) excelente $22,2 \%$ (6) bom.

A seguir são apresentados os principais resultados dos artigos analisados, tendo sido agrupados por semelhanças para melhor compreensão dos mesmos:

\section{Avaliação da estrutura das unidades de saúde para atenção puerperal}

Dos artigos avaliativos, três ${ }^{11,20,21}$ abordaram a avaliação da estrutura para atenção puerperal, todos realizados no Brasil. A atenção puerperal é prestada, principalmente, por médicos e enfermeiros ${ }^{11,20}$, com problemas principalmente na fixação e qualificação do médico na $\mathrm{APS}^{11}$. No estudo de Santos/SP foi avaliada a inserção do nutricionista, que se dá de forma insuficiente, estando presente em torno de $65 \%$ das unidades por apenas um ou dois períodos da semana ${ }^{21}$.
Quanto à avaliação da estrutura física e material os estudos encontraram que a maior parte das unidades de saúde dispunha de infraestrutura mínima para atenção às puérperas, como sala individual e disponibilidade de materiais, vacinas e medicamentos necessários para atenção à mulher no puerpério. Entretanto, uma das limitações para assistência é a disponibilização e manutenção da funcionalidade dos materiais ${ }^{11,20,21}$. Os estudos também apontaram pouca realização de ações de educação em saúde e espaço físico inadequado ou inexistente para esse fim ${ }^{11,20,21}$.

\section{Realização da consulta pós-parto: procura/oferta e motivos da não adesão}

Estudos que avaliaram a atenção ao pré-natal, conforme preconizado pelo Programa de Humanização no Pré-Natal e Nascimento (PHPN) no Brasil, mostraram, desde a sua implantação até a atualidade, que a recomendação de ter seis ou mais consultas de pré-natal, todos os exames básicos, o teste HIV, a 2a dose ou a de reforço ou a imunizante da vacina antitetânica, e consulta de puerpério é pouco cumprida ${ }^{20,22,23}$.

Quanto aos estudos brasileiros que avaliaram o indicador realização da consulta de puerpério 
até $42^{\circ}$ dias pós-parto identificou-se baixa realização, variando de $16,8 \%$ a $58 \%^{24-26}$. Foi identificada falta ou pouca flexibilidade agendamento prévio da consulta puerperal ${ }^{11,18}$ e na realização da Primeira Semana de Saúde Integral ${ }^{18,21}$. Os estudos apontam que em mais da metade dos casos é realizada a busca ativa de faltosas, porém não se constitui em prática sistemática para todas as puérperas $^{11,20,21,25}$.

Estudo realizado em 9 regiões mais pobres do Peru evidenciou que 58\% das mulheres referiram ter tido controle pós-parto dentro de 42 dias $^{27}$. Já, pesquisa realizada no Reino Unido, em 1995, apontou que $91 \%$ das mulheres compareceram para a consulta de puerpério seis semanas pós - parto $^{28}$.

A literatura, internacional e nacional, mostra como motivos para não adesão à consulta puerperal a falta de informação por parte dos profissionais das ações pós-parto ${ }^{4,5}$; falta de medicamentos e equipamentos; falta de qualificação profissional; barreiras culturais ${ }^{4}$; e percepção de que o cuidado pós-parto é para a criança ${ }^{4,5,7}$, e além disso, as ações educativas à puérpera ainda são realizadas de forma tradicional, o que também desestimula a participação das mesmas ${ }^{29}$.

$\mathrm{Na}$ Austrália evidenciou-se que os problemas relacionados a ocorrência da atenção pós-parto de forma organizada e com maior qualidade se dá pela falta de diretrizes consistentes e coesas para cuidados comunitários das novas mães e seus bebês ${ }^{5}$. Dois estudos australianos ${ }^{5,7}$ e um do Reino Unido ${ }^{28}$ identificaram que as puérperas valorizam e estão abertas aos cuidados primá$\operatorname{rios}^{5,7,28}$ desde que a consulta seja um momento para serem compreendidas, apoiadas e tranquilizadas, e são mais propensas a falar sobre suas necessidade quando questionadas sobre elas ${ }^{5,7}$.

\section{Abordagem da mulher na consulta puerperal}

Quanto às ações desenvolvidas por profissionais durante a consulta de puerpério, pesquisa do Reino Unido indica que em 93\% das mulheres tiveram exame abdominal e 70\% vaginal, entretanto muitas mulheres têm exames sem razão óbvia (como exame vaginal sem necessidade), enquanto outros testes que podem ser úteis para certas condições (como hemograma para mulheres com fadiga) são usados com pouca frequência ${ }^{28}$.

Estudos brasileiros apontam que as atividades mais comuns desenvolvidas no puerpério pelos profissionais de saúde, além do incentivo ao aleitamento materno, são orientações sobre os métodos de anticoncepção ${ }^{18,25}$, alimentação e nutrição ${ }^{18,21}$, e uso de sulfato ferroso ${ }^{18}$. Ações menos frequentes são realização de coleta de exame citopatológico de colo de útero ${ }^{24}$, exame clínico de mamas ${ }^{18,24}$, investigação sobre estado emocional da mulher, orientação quanto ao retorno às relações sexuais, avaliação de lóquios e exame da região genital ${ }^{18}$. Já, em estudo nacional que avaliou a atenção puerperal junto a adolescentes classificou essa como adequada para $43,2 \%$ das usuárias, sendo que as demais receberam assistência intermediária ou inadequada, considerando nessa avaliação a realização de consulta de revisão puerperal, visita domiciliar na primeira semana após o parto, avaliação clínico-ginecológica, orientação sobre planejamento familiar e orientação sobre aleitamento materno ${ }^{25}$. No Peru evidenciou-se que somente $38,5 \%$ das mulheres usam sulfato ferroso e 12,6\% vitamina A 30 dias após o parto, e 31\% haviam escolhido um método contraceptivo ${ }^{27}$.

Durante os primeiros 3 meses pós-parto, estudos australianos indicaram que $89 \%$ das mulheres têm problemas de saúde sexual, e, embora a maioria das mulheres tenha contato com profissionais de APS durante esse período, apenas $24 \%$ lembrou-se de ter sido questionada sobre o tema por médicos de clínica geral e $14 \%$ por enfermeiras ${ }^{30,31}$. As mulheres expressaram insatisfação com a qualidade dos cuidados de saúde primários pós-natais relatando uma forte mudança no foco da saúde da mãe para a saúde do bebê após o nascimento ${ }^{30}$.

\section{A visita domiciliar como ferramenta para atenção pós-parto}

Dos 42 estudos selecionados para a revisão, nove abordaram o tema visita domiciliar (VD) no pós-parto, sendo quatro nacionais ${ }^{18,21,25,32}$ e cinco internacionais ${ }^{33-37}$. Quatro estudos indicam baixa realização da $\mathrm{VD}^{18,21,25,33}$ e no Brasil a mesma ocorre com maior frequência quando se trata de unidade de saúde da família, realizadas principalmente por Agentes Comunitários de Saúde $(\mathrm{ACS})^{18,25}$. A VD é mais provável de ocorrer quando há acompanhamento da mulher durante o pré-natal pela equipe ${ }^{18,33}$.

Quanto ao impacto positivo de programas de VD durante o ciclo gravídico-puerperal, estudos internacionais apontaram para redução do tabagismo entre puérperas ${ }^{34}$, melhora nos cuidados e no desenvolvimento da criança ${ }^{36,37}$, maior apoio social e psicológico às mulheres e famílias ${ }^{37}$, e, 
para três estudos internacionais ${ }^{34-36}$ e dois nacionais ${ }^{21,32}$ foi identificado aumento do aleitamento materno exclusivo até seis meses. Para a realização de VD pós-parto faz-se necessária a composição de equipe multiprofissional, o que impacta na melhoria da qualidade da atenção e estreita vínculos entre equipe e mulheres/família ${ }^{21,37,38}$.

\section{Aleitamento materno}

Dos estudos revisados, o aleitamento materno se apresentou como a principal ação abordada pelos profissionais no Brasil, sendo um tema pouco investigado internacionalmente ao tratar de ações à mulher no puerpério em APS. Sete pesquisas brasileiras fizeram avaliação de impacto da prevalência desse, antes e após iniciativas de incentivo ao aleitamento materno (como "unidade básica amiga da amamentação" ou "rede amamenta Brasil"), identificando que tais ações contribuem para o aumento da taxa de aleitamento materno, tanto exclusivo até seis meses, como posterior a esse período ${ }^{19,32,39-41}$. Dois estudos não encontraram significância estatística em seus resultados, apesar de também terem evidenciado superioridade no percentual de aleitamento materno após a implantação da iniciativa ${ }^{42,43}$.

Pesquisa realizada em Francisco Morato/SP que avaliou os conhecimentos de profissionais de saúde sobre aleitamento materno, evidenciou que esses reconhecem a importância do aleitamento materno para o binômio mãe/filho, porém citam com mais facilidade os benefícios para a criança. Além disso, apesar de fazerem diversas orientações às mulheres, possuem conhecimento limitado sobre o tema ${ }^{44}$.

\section{Depressão pós-parto (DPP): manejo não especializado na APS}

A literatura internacional evidencia que programas de rastreamento da DPP no âmbito da APS podem melhorar o resultado de saúde mental das mulheres no período pós-parto ${ }^{45-49}$, mesmo em regiões de baixa renda e com tecnologias de baixo custo, conforme apontado por estudos chileno $^{47}$ e australiano ${ }^{50}$. A Edimburgh Post-Natal Depression Scale (EPDS), validada em diversos países, é reconhecida como a principal ferramenta para rastreamento da DPP por profissionais não especializados no âmbito da $\mathrm{APS}^{45-49,51}$.

Ressalta-se que enfermeiros de saúde pública estão bem posicionados para identificar e tratar mulheres deprimidas e encaminhá-las quando necessário. Um pequeno investimento na forma- ção de enfermeiros para identificar e tratar a DPP pode ter impactos positivos a longo prazo $^{45,48}$.

A investigação por um profissional de saúde sobre a saúde mental, passada ou atual das mulheres, está associada à busca de ajuda ao longo do período perinatal, conforme estudo australia$\mathrm{no}^{50}$. Na Dinamarca foi encontrado que puérperas que desenvolveram um episódio psiquiátrico apresentaram maiores taxas de consulta por profissionais de saúde antes, durante e após a gravi$\operatorname{dez}^{52}$.

Barreiras para tratamento da DPP, foram evidenciadas por estudo no Chile, como: falta de conhecimento das usuárias sobre a doença, conceituação negativa e rejeição das opções de tratamento disponíveis; suporte de rede deficiente e longos tempos de espera e falta de coordenação entre decisões clínicas e administrativas ${ }^{53}$.

Entre os estudos que abordaram o tema DPP, três são nacionais, e identificaram conhecimento insuficiente dos profissionais da APS para prevenir e tratar DPP, falta do uso de instrumento sistemático para rastreamento de DPP, e que o foco da atuação durante a gestação e pós-parto ainda é biológico ${ }^{54-56}$.

\section{Discussão}

A busca bibliográfica realizada no presente estudo iniciou com grande quantidade de referências (1.974), e 43 foram selecionadas para análise conforme critérios de inclusão e exclusão. A maior parte das referências que compuseram a amostra inicial da busca tratava de saúde da criança ou saúde da mulher em outros momentos da vida, não referindo-se ao puerpério.

Pode-se considerar que a literatura que aborda a atenção à mulher no puerpério no âmbito da APS é escassa, pois a maior parte dos estudos selecionados não fez uma abordagem integral da atenção puerperal, de modo que as evidências limitaram-se a temas pontuais de ações específicas, e estudos avaliativos restringiram-se apenas a avaliações de processo. Dos estudos que fizeram avaliação do processo do puerpério, apenas o de Silva et al. ${ }^{18}$ fez uma investigação mais ampla sobre as ações desenvolvidas no puerpério, enquanto os demais, em sua maioria, principalmente os brasileiros, restringiram-se a investigar número de consulta pós-parto e impacto de programas de incentivo ao aleitamento materno.

Houve aumento da produção científica nacional e internacional nos últimos 5 anos, indicando que a preocupação com a saúde da mulher 
no puerpério vem crescendo, possivelmente pela propulsão de estratégias que visam redução da morbimortalidade materna e infantil, propostas por exemplo pelos Objetivos de Desenvolvimento Sustentável, e adotadas por diversos países. Apesar do decréscimo mundial, nas últimas décadas, a mortalidade materna e infantil ainda continua sendo um problema de saúde pública, especialmente em países em desenvolvimento, assim como países desenvolvidos verificam necessidade de qualificação da atenção à mulher no pós-parto para redução da morbidade materna, o que também pode ter fomentado o aumento de estudos nos últimos anos.

A atenção puerperal prestada na APS, de acordo com os estudos analisados, predomina em indicadores de consulta pós-parto e aleitamento materno, em artigos nacionais, e programas de visita domiciliar e DPP sendo realizados principalmente em nível internacional. Outros aspectos da atenção como avaliação de estrutura física e materiais, profissionais de saúde, e demais atividades desenvolvidas no puerpério (orientações em geral, avaliação física, avaliação de vulnerabilidades sociais) são pouco investigadas.

No Brasil, as investigações se limitam ao puerpério imediato e tardio, abordando puerpério remoto apenas quando se trata de aleitamento materno, e este com foco na criança. Estudos internacionais trataram sobre puerpério remoto em no máximo dois anos e meio pós-parto ${ }^{34,36}$, com predominância de investigação quanto a $\mathrm{DPP}^{46-}$ 48,50-52. Enquanto muitas mulheres transitam pelo puerpério sem intercorrências, outras experienciam problemas significativos que podem persistir por semanas, meses ou mesmo por mais de 4 anos após o parto ${ }^{7,8}$, indicando necessidade de cuidado dessas mulheres ao longo do tempo.

Considerando que a saúde materna é sensível à $\mathrm{APS}^{2,3}$ e que esse ponto de atenção deveria prestar o cuidado à mulher de forma longitudinal, há necessidade de qualificar tais ações a fim de acompanhar a mulher em todas as fases do puerpério, inclusive no remoto, negligenciado não somente por políticas públicas e diretrizes clínicas ${ }^{3,6,57}$, mas também em estudos científicos. Ressalta-se que a maior parte da atenção no âmbito da APS são de baixo custo, e tem resultados significativos na redução da morbimortalidade materna com ações integradas e de acesso universal ${ }^{2,3}$.

Os resultados mostraram que em países em desenvolvimento (Brasil e Peru) a consulta puerperal ainda não ocorre em frequência satisfatória, e são países como esses que as taxas de mortalidade materna permanecem elevadas. Há necessidade de mudanças nas políticas de acesso à APS para as mulheres, seu companheiro e/ou familiares, durante esse período, o que poderia impactar na melhoria dos indicadores de morbimortalidade materna e infantil. A maioria dos estudos abordam o tema consulta puerperal apenas quanto a frequência de realização desta, pouco investigando a qualidade das mesmas.

O maior risco de mortalidade está durante o período pós-parto imediato e tardio, com maior taxa de morbimortalidade na primeira semana pós-parto, o que torna esse um momento crítico para a mulher e seu filho. A consulta pós-parto é uma intervenção primordial para redução da morbimortalidade materna, por meio da prevenção, detecção precoce e tratamento de complicações, e prestação de aconselhamento sobre contracepção ${ }^{4,5}$. Entretanto, essa ação não parece proporcionar uma oportunidade para mulheres terem suas necessidades de saúde atendidas, seja pela má qualidade da assistência ${ }^{28}$, ou, pela baixa frequência de realização dessas consultas ${ }^{23}$.

Além da baixa frequência de realização da consulta puerperal, os resultados dos estudos sobre o tema "abordagem da mulher na consulta de puerpério” indicam incipiência e precariedade na assistência prestada na APS, com atenção fragmentada, biologicista, atenção prioritária à criança, foco no papel de "mãe", não na mulher e suas necessidades, tanto em estudos nacionais como internacionais.

Diretrizes clínicas internacionais ${ }^{3,9,57,58}$ orientam a prestação da atenção à mulher no puerpério prioritariamente por profissionais da APS, por meio do cuidado integral e coordenado, valorizando as necessidades de saúde da mulher, com estímulo a autonomia e cuidado de si e da criança. Tais diretrizes são baseadas em alto grau de evidências científicas, e primam pela qualidade da prestação da assistência, aspecto esse que precisa ser melhor investigado nos estudos que tratam da consulta puerperal e melhor implementado na prática assistencial à mulher no puerpério no contexto da APS nos diferentes cenários do Brasil e internacionalmente.

A VD é um importante momento para a equipe de APS detectar mudanças físicas e emocionais precocemente ${ }^{9}$, desenvolver ações educativas, identificar riscos e vulnerabilidades da família, estabelecer vínculo profissional-usuário e potencializar a proteção à saúde da mulher e da criança no pós-parto ${ }^{18}$.

Sobre a referida estratégia de assistência à saúde, estudos internacionais fizeram análise de programas de VD e seus impactos, demonstrando a 
importância dessa para a atenção ao puerpério. Já estudos brasileiros limitam-se a investigar frequência de visitas e de forma incipiente as ações realizadas em VD, especialmente durante a primeira semana pós-parto, e o principal profissional a desempenhar essa função é o ACS, o qual também atua com foco na criança e atenção biologicista.

Sobre o aleitamento materno, apesar de ser um indicador de saúde da mulher no pós-parto, os estudos ainda abordam o tema com maior ênfase na criança. Esse tema, junto com o indicador número de consultas pós-parto, foram os que mais tiveram ênfase em estudos realizados no Brasil, com análise predominantemente quantitativa, indicando lacuna de investigações da qualidade do cuidado prestado na APS.

Inúmeros são os benefícios do aleitamento materno para a saúde da mulher ${ }^{19}$, e as principais dificuldades para a manutenção deste surgem nos primeiros meses pós-parto, sendo que a APS se constitui em um ambiente incentivador dessa prática ${ }^{41}$, considerando sua proximidade à mulher e seu papel prioritário na prestação da atenção puerperal ${ }^{9}$.

Em relação ao tema atenção à DPP identificado nos artigos analisados, foi amplamente investigado especialmente em estudos internacionais. A EPDS apareceu como importante ferramenta para rastreamento de DPP por profissionais de APS em países em desenvolvimento, a fim de assegurar a detecção precoce e a intervenção terapêutica adequada nos casos de DPP ${ }^{51}$. Já estudos nacionais apontam para a atenção "empírica” a DPP, com profissionais despreparados para rastreamento, prevenção e tratamento do referido $\operatorname{agravo}^{54-56}$.

A DPP é um fenômeno internacional, considerada a complicação mais comum no pós-parto. Vista como um problema de saúde pública, cuja prevalência varia de 10 a $15 \%$ em países desenvolvidos, atingindo até $40 \%$ em países em desenvolvimento ${ }^{45,53,56}$, tal agravo afeta a qualidade de vida da mulher, e o vínculo com o recém-nascido assim como seu desenvolvimento ${ }^{53,54,56}$.

Os profissionais de saúde da APS estão bem posicionados para fornecer apoio psicossocial a todas as mulheres no período perinatal e realizar abordagens preventivas entre as mulheres que podem estar com maior probabilidade de desenvolver DPP ou distúrbios relacionados, com particular importância no puerpério imediato $^{9,58}$. Esses profissionais devem ter papel ativo no rastreamento e seleção do tratamento de DPP, considerando fatores contextuais, como cultura, condição socioeconômica entre outros ${ }^{9,58}$.
As limitações encontradas para a realização da presente revisão se referiram à ampla gama de descritores utilizados, resultando em um número grande de artigos para ser realizada a leitura de títulos e resumos. Entretanto, essa estratégia foi necessária, devido a escassez de estudos que usam como descritor o período pós-parto, sendo superada tal limitação com a leitura minuciosa dos títulos e resumos, garantindo seleção dos estudos que abordassem a questão norteadora. Ainda, observou-se uma variedade de métodos de pesquisa utilizados para tratar das ações de programas de puerpério, o que impede a análise comparativa mais acurada dos resultados alcançados. Como qualquer tipo de estudo a revisão integrativa apresenta vantagens e limitações do próprio método, devendo haver cuidado no que tange às conclusões relacionadas aos achados. Entretanto, os autores destacam a potencialidade do método empregado, visto que o mesmo tem validade entre experts na área de revisão.

\section{Considerações finais}

Os resultados indicam que existe um leque de ações a serem desempenhadas pela APS para assistir à mulher no puerpério, as quais são passíveis de realização com o uso de tecnologias leves e de baixo custo, e que esse ponto de atenção é primordial para auxiliar na redução da morbimortalidade materna, por meio do aconselhamento e apoio para a recuperação da gravidez e nascimento, identificação precoce e gestão adequada das necessidades de saúde física e emocional.

Apesar da reconhecida importância da atenção puerperal, estudos brasileiros, em sua maioria limitam-se a investigar número de consulta pós-parto, número de visitas domiciliares e impacto de programas de incentivo ao aleitamento materno, negligenciando a integralidade da atenção pós-parto às mulheres na APS.

Estudos nacionais e internacionais indicam que a atenção pós-parto ainda tem como foco o cuidado ao recém-nascido e são restritos, em sua maioria, ao puerpério imediato e tardio, havendo necessidade de melhorias na implementação dos cuidados primários à saúde da mulher, especialmente no que tange à coordenação e longitudinalidade do cuidado, o que evidencia necessidade de melhoria na qualidade da atenção à mulher no puerpério na APS.

Ressalta-se que a DPP foi alvo de inúmeros estudos na literatura internacional, dada a sua alta prevalência e problema de saúde pública, e 
evidenciou alto potencial dos profissionais da APS para atuar na prevenção, rastreamento e tratamento do referido agravo. Já no Brasil, esse se constitui em tema pouco estudado e digno de atenção de políticas públicas no pós-parto, com resultados que apontam para falta de qualificação dos profissionais da APS para atenção à DPP.

A partir da presente revisão foram encontradas lacunas de conhecimento no que tange a temática abordada, com necessidade de estudos mais robustos, que tratem da atenção integral à mulher, assim como aspectos da qualidade do cuidado prestado na APS. Além disso, verifica-se a necessidade de pesquisas avaliativas, as quais permitem, com abordagem qualitativa e quantitativa, compreender a implantação da atenção puerperal na APS e a relação dos resultados encontrados com o programa de atenção puerperal.

É importante destacar que a investigação do puerpério pode identificar uma amplitude de atividades a serem desenvolvidas e consideradas na APS, tanto no que se refere à estrutura física, material, humana, assim como qualificação profissional, planejamento e gestão do programa, além de fatores externos como aspectos culturais da mulher atendida. Assim, o presente estudo serve de subsídio tanto para nortear a elaboração e melhoria das ações à mulher no puerpério na APS por parte de profissionais e gestores, como para direcionar a elaboração de estudos empíricos que possam investigar a integralidade da atenção puerperal.

\section{Colaboradores}

T Baratieri trabalhou na concepção do manuscrito, metodologia, redação, e aprovação da versão final a ser publicada. S Natal trabalhou na concepção do manuscrito, metodologia, revisão crítica, e aprovação da versão final. 


\section{Referências}

1. World Health Organization (WHO). Global Health Observatory (GHO) data. Women and health [Internet] 2017 [cited 2017 Jun 10]. Available from: http:// www.who.int/gho/en/

2. Bhutta ZA, Cabral S, Chan C-w, Keenan WJ. Reducing maternal, newborn, and infant mortality globally: An integrated action agenda. Obstet Gynecol Int J 2012; 119(Supl. 1):13-17.

3. World Health Organization (WHO). WHO recommendations on Postnatal care of the mother and newborn. Geneva: WHO Library Cataloguing-in-Publication Data; 2013.

4. Nabukera SK, Witte K, Muchunguzi C, Bajunirwe F, Batwala VK, Mulogo EM, Farr C, Barry S, Salihu HM. Use of postpartum health services in rural Uganda: knowledge, attitudes, and barriers. J Community Health 2006; 31(2):84-93.

5. Brodribb W, Zadoroznyj M, Dane A. The views of mothers and GPs about postpartum care in Australian general practice. BMC Fam Pract 2013; 14(1):1-9.

6. Brasil. Ministério da Saúde (MS), Instituto Sírio-Libanês de Ensino e Pesquisa. Protocolos da Atenção Básica: Saúde das Mulheres. Brasília: MS; 2016.

7. Hartley S, Sutherland G, Brown S, Yelland J. 'You're more likely to tell the GP if you're asked': women's views of care from general practitioners in the first postpartum year. Aust J Prim Health 2012; 18(4):308312.

8. Gartland D, MacArthur C, Woolhouse H, McDonald E, Brown S. Frequency, severity and risk factors for urinary and faecal incontinence at 4 years postpartum: a prospective cohort. Int J Obstet Gynecol 2016; 123(7):1203-1211.

9. National Institute for Health and Care Excellence (NICE). Antenatal and postnatal mental health: clinical management and service guidance. London: NICE; 2015.

10. Correa MS, Feliciano KV, Pedrosa EN, Souza AI. Women's perception concerning health care in the post-partum period: a meta-synthesis. Open J Obstet Gynecol 2014; 4:416-426.

11. Oliveira DC, Teixeira RC, Tomiyoshi JT, Correa ACdP, Mandu ENT. Estrutura organizacional da atenção pós-parto na estratégia saúde da família. Esc Anna Nery Rev Enferm 2013; 17(3):446-554.

12. Corrêa MSM, Feliciano KVO, Pedrosa EN. Souza AI. Acolhimento no cuidado à saúde da mulher no puerpério. Cad Saude Publica 2017; 33(3):1-12.

13. Mendes KDS, Silveira RCCP, Galvão CM. Revisão integrativa: método de pesquisa para a incorporação de evidências na saúde e na enfermagem. Texto \& contexto enferm 2008; 17(4):758-764.

14. Stufflebeam DL. Program Evaluation Models Metaevaluation Checklist (Based on The Program Evaluation Standards). Evaluation Checklists Project [Internet] 1999 [cited 2017 May 15]. Available from: http:// dmeforpeace.org/learn/program-evaluations-models-metaevaluation-checklist
15. Critical Appraisal Skills Programme (Casp). Programme AS. Making sense of evidence. CASP Checklists [Internet] 2017 [ cited 2017 May 15]. Available from: http://www.casp-uk.net/casp-tools-checklists

16. Stetler CBMD, Rucki S, Broughton S, Corrigan B, Fitzgerald J, Giuliano K, Havener P, Sheridan A. Utilization-focused integrative reviews in a nursing service. Appl Nurs Res 1998; 11(4):195-206.

17. Ursi E. Prevenção de lesões de pele no perioperatório: revisão integrativa da literatura [dissertação]. Ribeirão Preto: Universidade de São Paulo; 2005.

18. Silva LLB, Feliciano KVO, Oliveira LNFP, Pedrosa EN, Corrêa MSM, Souza AI. Cuidados prestados à mulher na visita domiciliar da "Primeira Semana de Saúde Integral”. Rev Gaucha Enferm 2016; 37(3):1-8.

19. Rito RV, Oliveira MI, Brito Ados S. Degree of compliance with the ten steps of the Breastfeeding Friendly Primary Care Initiative and its association with the prevalence of exclusive breastfeeding. J Pediatr (Rio J) 2013; 89(5):477-484.

20. Parada CMG. Avaliação da assistência pré-natal e puerperal desenvolvidas em região do interior do Estado de São Paulo em 2005. Rev. bras. saúde mater. infant 2008; 8(1):113-124.

21. Laporte-Pinfildi ASC, Zangirolani LTO, Spina N, Martins PA, Medeiros MAT. Atenção nutricional no pré-natal e no puerpério: percepção dos gestores da Atenção Básica à Saúde. Rev nutr 2016; 29(1):109-123.

22. Serruya SJ, Lago T, Cecatti JG. Avaliação preliminar do programa de humanização no pré-natal e nascimento no Brasil. Rev bras ginecol obstet 2004; 26(7):517-525.

23. Corrêa MD, Tsunechiro MA, Lima MOP, Bonadio IC. Avaliação da assistência pré-natal em unidade com estratégia saúde da família. Rev Esc Enferm USP 2014; 48(n. esp.):23-31.

24. Santos SR, Cunha AJLA, Gamba CM, Machado FG, Leal Filho JMM, Moreira NLM. Avaliação da assistência à saúde da mulher e da criança em localidade urbana da região Sudeste do Brasil. Rev Saude Publica 2000; 34(3):266-271.

25. Vilarinho LM, Nogueira LT, Nagahama EEI. Avaliação da qualidade da atenção à saúde de adolescentes no pré-natal e puerpério. Esc Anna Nery Rev Enferm 2012; 16(2):312-319.

26. Hass CN, Teixeira LB, Beghetto MG. Adequabilidade da assistência pré-natal em uma estratégia de saúde da família de Porto Alegre-RS. Rev Gaucha Enferm 2013; 34(3):22-30.

27. Velásquez Hurtado JE, Solís Alcedo L, Vigo Valdez WE, Rosas Aguirre AM, Giusti Hundskopf P, Alfaro Fernandez P, Cabrera Arredondo H. Evaluación de las prácticas de cuidado materno infantil en áreas con pobreza extrema del Perú, 2012. Rev Peru Med Exp Salud Publica 2014; 31(2):243-253.

28. Bick DE, MacArthur C. Attendance, content and relevance of the six week postnatal examination. Midwifery 1995; 11(2):69-73. 
29. Guerreiro EM, Rodrigues DP, Queiroz ABA, Ferreir MA. Educação em saúde no ciclo gravídico-puerperal: sentidos atribuídos por puérperas. Rev Bras Enferm 2014; 67(1):13-21.

30. Woolhouse H, McDonald E, Brown SJ. Changes to sexual and intimate relationships in the postnatal period: women's experiences with health professionals. Aust J Prim Health 2014; 20(3):298-304.

31. McDonald E, Woolhouse H, Brown SJ. Consultation about sexual health issues in the year after childbirth: a cohort study. Birth 2015; 42(4):354-361.

32. Graça LCC, Figueiredo MCB, Conceição MTCC. Contributions of the nursing intervention in primary healthcare for the promotion of breastfeeding. Rev Lat Am Enfermagem 2011; 19(2):429-436.

33. Sitrin D, Guenther T, Murray J, Pilgrim N, Rubayet S, Ligowe R, Pun B, Malla H, Moran A. Reaching mothers and babies with early postnatal home visits: the implementation realities of achieving high coverage in large-scale programs. PLoS One 2013; 8(7):e68930.

34. Mejdoubi J, van den Heijkant SC, van Leerdam FJ, Crone M, Crijnen A, HiraSing RA. Effects of nurse home visitation on cigarette smoking, pregnancy outcomes and breastfeeding: a randomized controlled trial. Midwifery 2014; 30(6):688-695.

35. Bashour HN, Kharouf MH, AbdulSalam AA, El Asmar K, Tabbaa MA, Cheikha SA. Effect of postnatal home visits on maternal/infant outcomes in Syria: a randomized controlled trial. Public Health Nurs 2008; 25(2):115-125.

36. Robling M, Bekkers M-J, Bell K, Butler CC, Cannings-John R, Channon S, Martin BC, Gregory JW, Hood K, Kemp A, Kenkre J, Montgomery AA, Moody G, Owen-Jones E, Pickett K, Richardson G, Roberts ZE, Ronaldson S, Sanders J, Stamuli E, Torgerson D. Effectiveness of a nurse-led intensive home-visitation programme for first-time teenage mothers (Building Blocks): a pragmatic randomised controlled trial. Lancet 2016; 387(10014):146-155.

37. Saïas T, Lerner E, Greacen T, Simon-Vernier E, Emer A, Pintaux E, Guédeney A, Dugravier R, Tereno S, Falissard B, Tubach F; CAPEDP Study Group, Revah-Levy A. Evaluating fidelity in home-visiting programs a qualitative analysis of 1058 home visit case notes from 105 families. PloS One 2012; 7(5):e36915.

38. Laporte-Pinfildi ASC, Medeiros MAT. Nutritional care during prenatal and postpartum periods: A report of experiences in a city on São Paulo's coast. Rev nutr 2016; 29(6):947-961.

39. Cardoso LO, Vicente AS, Damião JJ, Rito RV. The impact of implementation of the Breastfeeding Friendly Primary Care Initiative on the prevalence rates of breastfeeding and causes of consultations at a basic healthcare center. J Pediatr 2008; 84(2):147-153.

40. Alves ALN, Oliveira MIC, Moraes JR. Iniciativa Unidade Básica Amiga da Amamentação e sua relação com o aleitamento materno exclusivo. Rev Saude Publica 2013; 47(6):1130-1140.
41. Passanha A, Benicio MHD, Venancio SI, Reis MCG Implantação da Rede Amamenta Brasil e prevalência de aleitamento materno exclusivo. Rev Saude Publica 2013; 47(6):1141-1148.

42. Almeida CC, Scochi MJ, Souza RKT, Carvalho WO. Prevalência de aleitamento materno antes e após a implantação de um programa de redução de morbimortalidade infantil, no município de Campo Mourão (PR). Cien Saude Colet 2010; 15(2):575-583.

43. Brandao DS, Venâncio SI, Giugliani ER. Association between the Brazilian Breastfeeding Network implementation and breastfeeding indicators. J Pediatr 2015; 91(2):143-151.

44. Ciconi RCV, Venancio SI, Escuder MML. Avaliação dos conhecimentos de equipes do Programa de Saúde da Família sobre o manejo do aleitamento materno em um município da região metropolitana de São Paulo. Rev bras saúde mater infant 2004; 4(2):193-202.

45. Leung SS, Leung C, Lam T, Hung S, Chan R, Yeung T, Miao M, Cheng S, Leung SH, Lau A, Lee DT. Outcome of a postnatal depression screening programme using the Edinburgh Postnatal Depression Scale: a randomized controlled trial. J Public Health 2010; 33(2):292301.

46. Milgrom J, Ericksen J, Negri L, Gemmill AW. Screening for postnatal depression in routine primary care: properties of the Edinburgh Postnatal Depression Scale in an Australian sample. Aust N Z J Psychiatry 2005; 39(9):833-839.

47. Rojas G, Fritsch R, Solis J, Jadresic E, Castillo C, González M, Guajardo V, Lewis G, Peters TJ, Araya R. Treatment of postnatal depression in low-income mothers in primary-care clinics in Santiago, Chile: a randomised controlled trial. Lancet 2007; 370(9599):1629-1637.

48. Glavin K, Smith L, Sørum R, Ellefsen B. Redesigned community postpartum care to prevent and treat postpartum depression in women-a one-year follow-up study. J Clin Nurs 2010; 19(21-22):3051-3062.

49. Segre LS, O’Hara MW, Brock RL, Taylor D. Depression screening of perinatal women by the Des Moines Healthy Start Project: program description and evaluation. Psychiatr Serv 2012; 63(3):250-255.

50. Reilly N, Harris S, Loxton D, Chojenta C, Forder P, Austin MP. The impact of routine assessment of past or current mental health on help-seeking in the perinatal period. Women Birth 2014; 27(4):e20-e7.

51. Abiodun O. Postnatal depression in primary care populations in Nigeria. Gen Hosp Psychiatry 2006; 28(2):133-136

52. Munk-Olsen T, Pedersen HS, Laursen TM, FengerGrøn M, Vedsted P, Vestergaard M. Use of primary health care prior to a postpartum psychiatric episode. Scand J Prim Health Care 2015; 33(2):127-133.

53. Rojas. Barreras de acceso a tratamiento de la depresión posparto en centros de atención primaria de la Región Metropolitana: un estudio cualitativo. Rev Med Chile 2015; 143(4):424-432. 
54. Meira B, Pereira P, Silveira M, Gualda D, Santos Jr H. Desafios para profissionais da atenção primária no cuidado à mulher com depressão pós-parto. Texto \& Contexto Enferm 2015; 24(3):706-712.

55. Felix TA, Siqueira DA, Ximenes Neto FRG, Nascimento KV, Mira QLM. Actuación de enfermería frente a la depresión postparto en las consultas de puericultura. Enferm Glob 2013; 12(29):404-419.

56. Santos Junior HPO, Gualda DMS, Silveira MFA, Hall WA. Postpartum depression: the (in) experience of Brazilian primary healthcare professionals. J Adv Nurs 2013; 69(6):1248-1258.

57. National Institute for Health and Care Excellence (Nice). Postnatal care up to 8 weeks after birth [Internet] 2015 [cited 2017 Out 27]. Available from: nice. org.uk/guidance/cg37

58. Austin MP, Highet, Guidelines Expert Advisory Committee. Clinical practice guidelines for depression and related disorders: anxiety, bipolar disorder and puerperal psychosis, in the perinatal period. A guideline for primary care health professionals. Melbourne: Beyondblue; 2011.

Artigo apresentado em 14/08/2017

Aprovado em 11/04/2018

Versão final apresentada em 13/04/2018 